\title{
Autodrive Land Vehicle Control by Using PID
}

\author{
I Swadexi, U Rudi, N Heriansyah \\ Electrical Engineering Field, Institut Teknologi Sumatera, Lampung, Indonesia
}

\begin{abstract}
In towards industry 4.0, the autodrive vehicle is needed to give people relaxed driving. There are many research in autodrive vehicle such as waymo-the Google driving car project and Tesla Self Driving Autopilot. In This paper is presented about Autodrive Land Vehicle (ALV) control by using PID. The autodrive Land vehicle can drive autonomously by using GPS Information such as Latitude and longitude to navigate in an area. The vehicle is controled to follow the given waypoint that set by operator on ground control station. PID control is used to control steering of the vehicle's front wheel and to control the heading of the vehicle. From the Implementation result, it is obtained that the vehicle could track the given waypoint with small error.
\end{abstract}

\section{Introduction}

Currently, unmanned vehicle technology has been widely used in surveillance, search and rescue purposes. In general, unmanned vehicles can be classified into three types, air rides, unmanned sea rides and land rides. The focus of this research is the automatic moving unmanned land vehicle (Autonomous Land Vehicle / ALV), the unmanned land vehicle in the future will have several application uses, some of which are:

1. Transportation, for example buses, private cars and bicycles

2. The vehicle with functions for example is automatic garbage truck, industrial robot

3. Agricultural vehicles, for example tractors, rice cutters and rice growers.

Along with the rapid technology, unmanned land vehicles are being developed to be able to move automatically (autonomous) so that operators only need to provide trajectory targets that must be passed and actions that must be carried out by the vehicle. Several studies have been carried out to be able to make unmanned land vehicles able to move autonomously, including using artificial intelligence on computer vision to be able to see the road and obstacles around the vehicle, besides that the vehicle is also equipped with a Global Positioning System (GPS) sensor. GPS that can be used for public has the disadvantage of a position error rate in the horizontal plane of $\pm 2 \mathrm{~m}$, this error value is large enough for a vehicle to navigate properly autonomously. This research will apply the equations of the land vehicle kinematics model to correct GPS information errors in order to have errors close to zero, to track position, use Proportional Integral and Derivative (PID) control methods, where this method has been widely applied in various fields both in autonomous systems and in industry.

A lot of research related to unmanned vehicles has been done, an example is in [1] which discusses modeling and control of autonomous quadrotor which is one type of unmanned aerial vehicle, the control method used in the study is the PID method. Position tracking research on unmanned air rides was also carried out on [2], [3]. In [4] examined the unmanned underwater vehicle control system, where this vehicle is an autonomous submarine vehicle, the method used in this research is a multi input and multi output (MIMO) Sliding control mode. Similar studies of unmanned underwater rides were also carried out on [5], [6] and [7]. 
There are also many researches on unmanned land vehicles, such as in [8] which discuss the problem of autonoous land vehicles or unmanned land vehicles using the Model Ribbon, in [9] research using $\mathrm{H} \infty$ filters and extreme learning machines (ELM) to correct errors GPS, while in [10] using the inertial measurement unit (IMU) to correct information provided by GPS by utilizing angular velocity and vehicle acceleration information. In [11] using artificial intelligence in Computer vision for ALV mobile robots to be able to walk to recognize the road area.

From the above studies, it can be seen that a lot of research on Autonomous vehicles is currently being carried out, and there are also many studies on unmanned land rides, especially on the correction of GPS information, this shows that GPS correction can be done using the addition of sensors, artificial intelligence and control system.

\section{Matematics Model of Vehicle}

This modeling aims to obtain mathematical equations from the vehicle, where the mathematical equations of this vehicle will be used to correct information errors provided by GPS. The vehicle used is a prototype toy car as shown in Figure 1. This vehicle has a steering navigation system, where to make two turns the front wheels will form an angle.

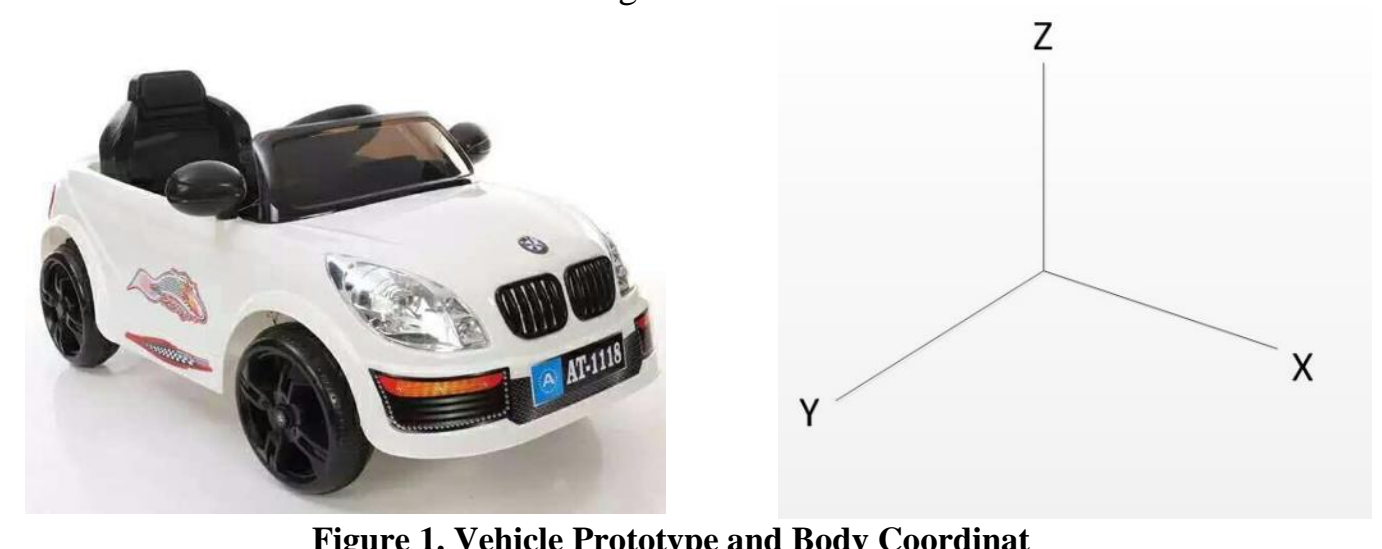

Figure 1. Vehicle Prototype and Body Coordinat

With the vehicle model as in the picture above, the mathematical equation will be obtained as stated in Equation (3.1):

$$
\begin{aligned}
& \dot{x}=u \cos \theta \\
& \dot{y}=u \sin \theta \\
& \dot{\theta}=\frac{u}{L} \tan \emptyset \\
& \text { Where : }
\end{aligned}
$$

$x, y$ : vehicle coordinates on the $\mathrm{x}$ and $\mathrm{y}$ axis of the vehicle.

$\theta$ : vehicle angles on earth coordinates

$\emptyset$ : steering wheel angle

$L$ : length of vehicle in $\mathrm{x}$ body coordinate

$u$ : control input

\section{Control Methods}

Proportional, Integral and Derivative (PID) control methods are the most widely used control methods in the industrial world, this is because this PID method can be used easily and has a lightweight computing process, so that almost all control system devices such as microcontrollers and computers can use This PID method, besides that the PID method also has the ability to make the system more 
robust against interference, this is because there is an Integrator controller in the PID method [12]. The PID equation, expressed in the following equation:

$$
\begin{aligned}
& u_{p}=K_{p} * e \\
& u_{d}=K_{d} \frac{d e}{d t} \\
& u_{i}=K_{i} \int e d t \\
& u_{p i d}=u_{p}+u_{i}+u_{d}
\end{aligned}
$$

Where $\mathrm{Kp}$ is a gain or proportional constant, $\mathrm{Ki}$ is the integral constant and $\mathrm{Kd}$ is the Derivative constant.

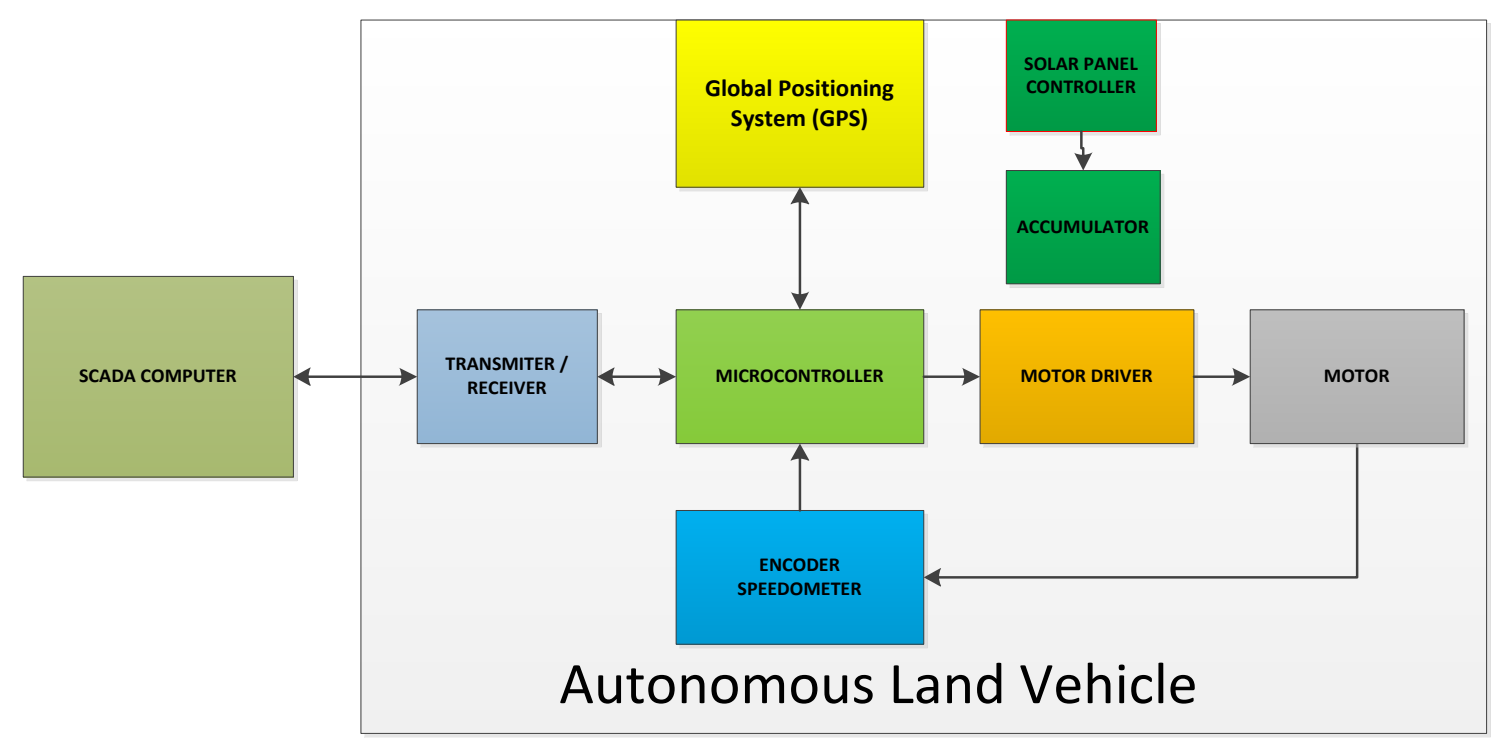

Figure 2. Diagram Block of Real Implementation System

\section{Result and Discussion}

\subsection{Simulation Result}

Before we implementing our vehicle by using real controller. We are trying to simulate the vehicle controller by using computer simulation. The vehicle's Mathematical model is derived in Equation (1). This mathematical model is used as vehicle or plant in computer simulation, then the controller is using PID to control Steering Angle of Vehicle, the result of computer simulation is XY Axis graph. The Diagram Block of vehicle control system is shown in Figure 3. 


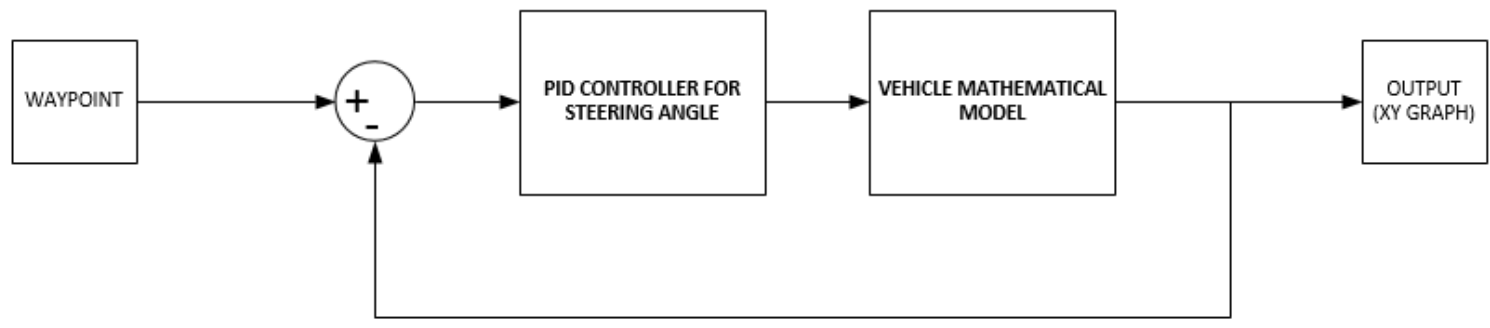

Figure 3. Diagram Block of Computer Simulation System

The simulation result is using XY plot, the XY coordinate in real implementations can be considered as longitude - latitude of earth coordinate. The waypoint coordinate that to be tracked by vehicle model from initial postion $\mathrm{XY}=(0,0)$ is $\mathrm{WP} 1=(10,10)$; WP2 $=(10,-10)$; WP3 $=(-10,-10) ; \mathrm{WP} 4=(-10,10)$ and return to WP1. The simulation result is shown in figure 4.

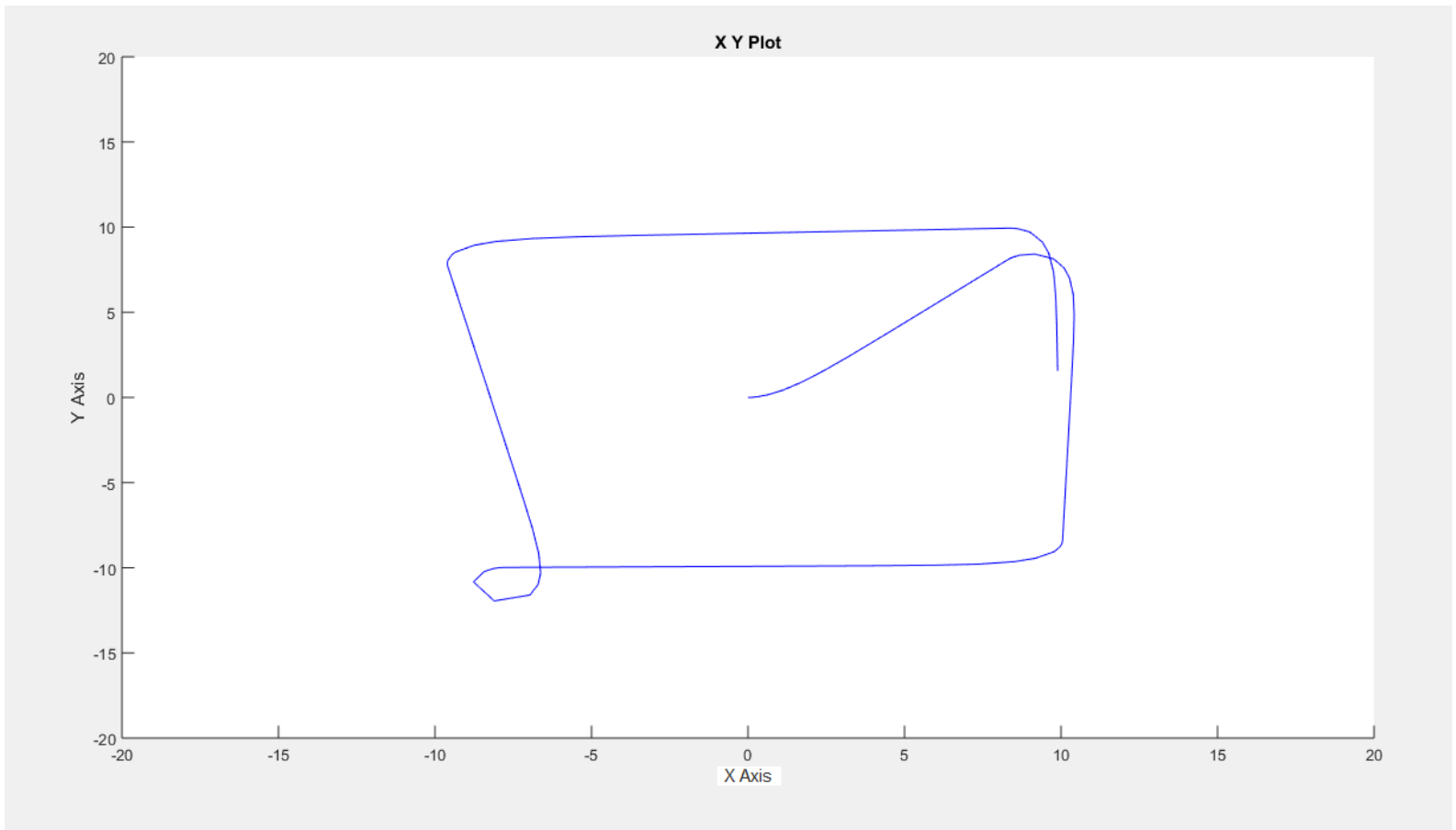

Figure 4. Computer simulation result

From computer simulation result that shown in Figure 4. We can see that vehicle start from initial position to first waypoint and then turn right to the next waypoint and return successfully to first waypoint. From this simulation result show that the PID control is succesfully control the vehicle to track the given waypoint.

\subsection{Implementation Result}

The prototype of land vehicle is shown in Figure 5. The vehicle is equiped with electronic devices such as Arduino microncontroler, Motor driver and Sensors. To control the vehicle to track the given coordinate, we are using motor driver to rotate the steering motor. This motor driver can control the direction of motor and control speed by using PWM pulse. 


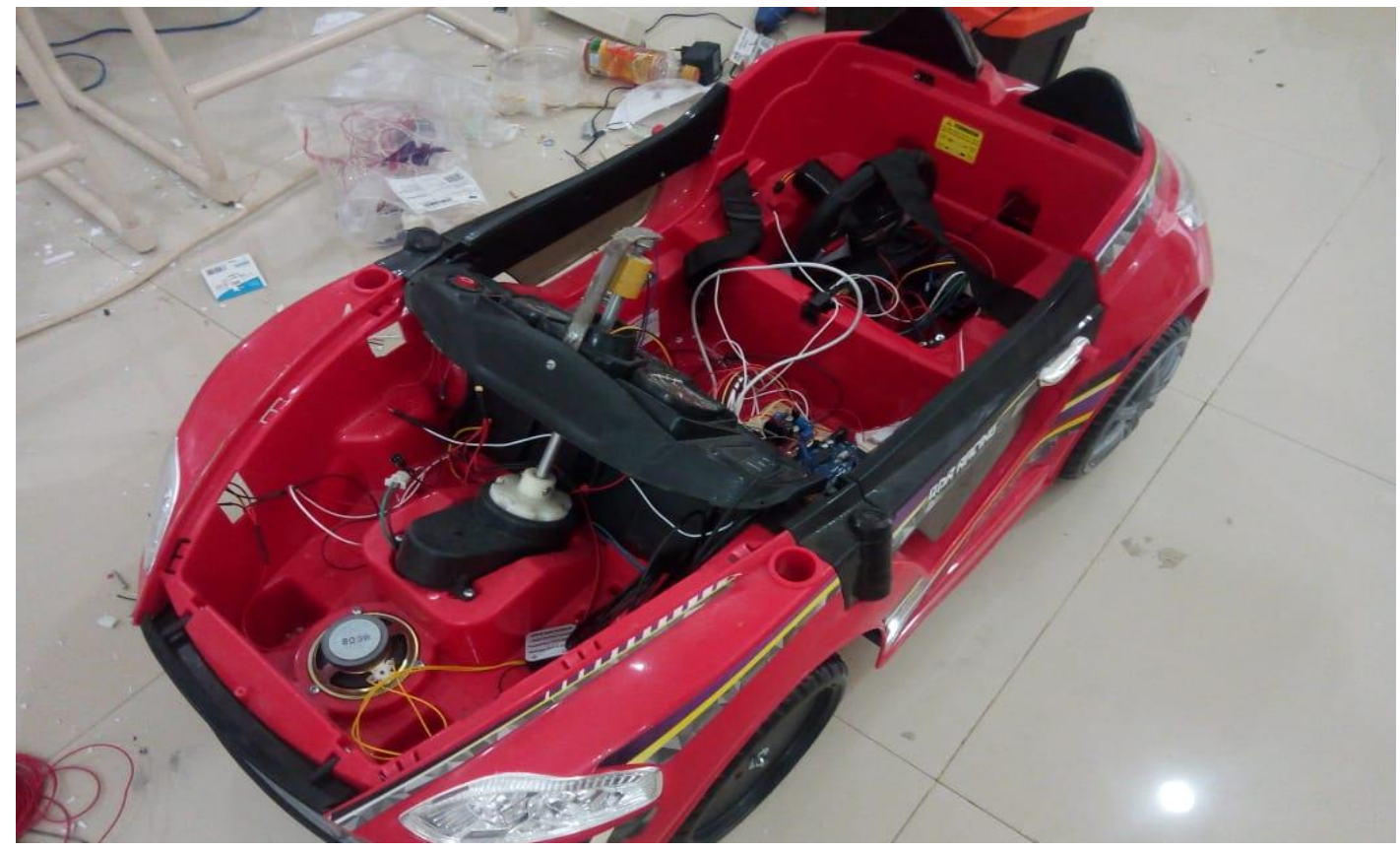

Figure 5. The Vehicle Prototype

The Implementation result is shown in Figure 5. where the vehicle is located at one place then the single waypoint is located at the other place where the distances is about 30 meters. To monitor the vehicle, internet of things is used to send data to webserver and using web to monitor the vehicle moving. In figure 5 shown that vehicle was move from top to bottom.

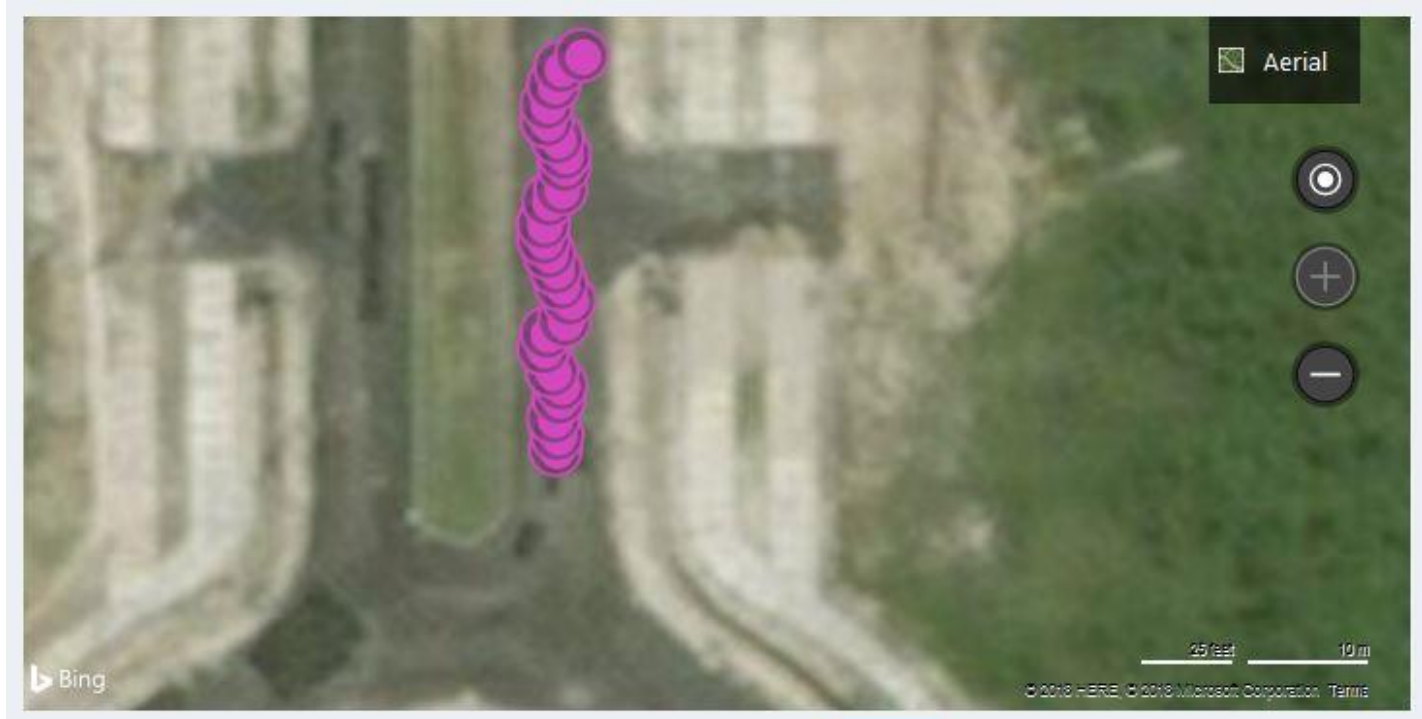

Figure 6. Implementation Result shown in Bing Maps 


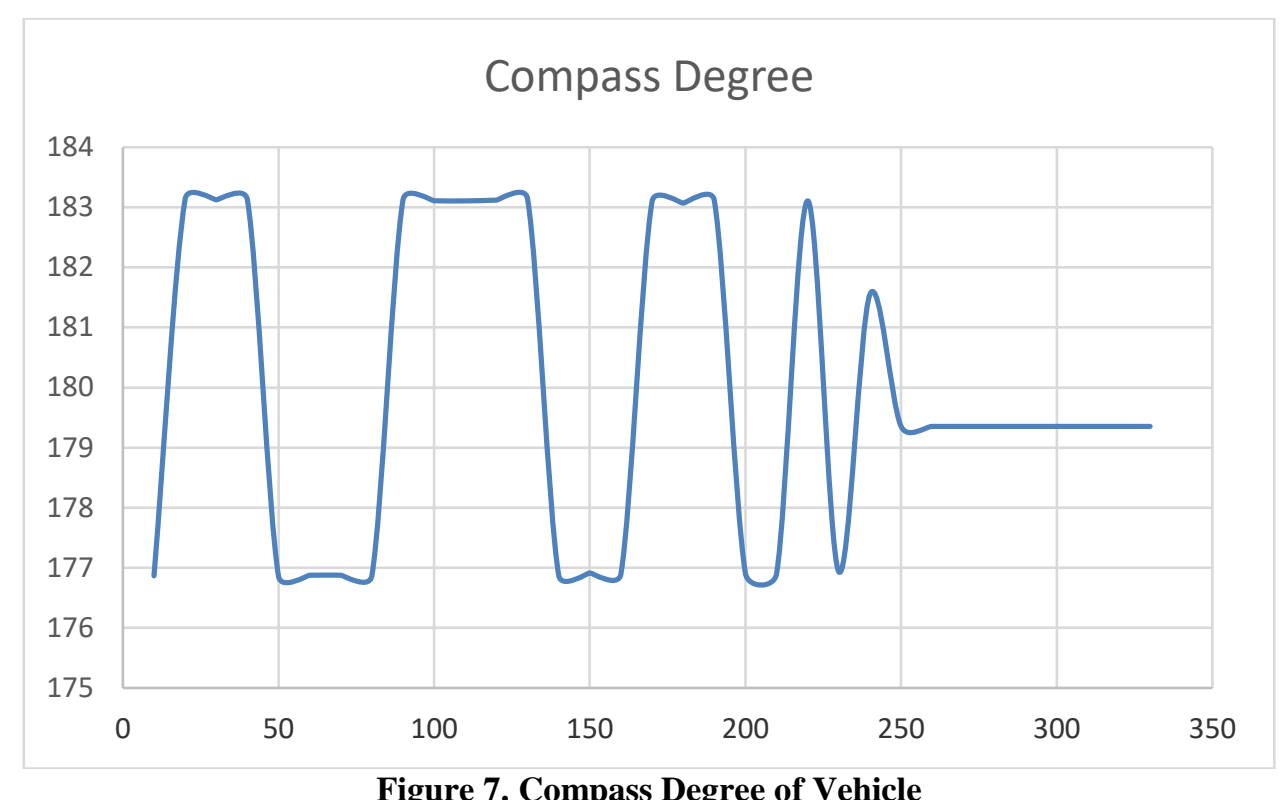

Figure 7. Compass Degree of Vehicle

The vehicle heading angle is shown in Figure 6, heading angle is obtained when vehicle moving to track the given waypoint in Figure 5. From the result shown that vehicle heading angle is still osilated and cause the vehicle not smooth in driving. This can be caused by choosing gain of PID not accuratelly and also from GPS sensors that provide not accurate and not precission coordinate.

\section{Conclussion}

This is a research about how to control land vehicle using PID, the final target of this research is to obtain the autonomous vehicle such as buses and garbage vehicle that working in Campus. In this research we are using vehicle prototype that modified from toys that powered electrically. To control autonomously we are using PID method that control steering angle of vehicle. From the simulation result it is obtained that vehicle can track the given waypoints succesfully. From the implementation result that monitored using Internet of Things the vehicle can track the single waypoint but still need to be improved to driving smoothly. The future research is PID gain is need to be choosen analitically in order obtain the best PID gain. And Also need control method to improved the GPS information.

\section{Acknowledgments}

Thanks to Institut Teknologi Sumatera (ITERA), this research is funded by ITERA Smart Mandiri Research.

\section{References}

[1] G. Jithu and J. P R, "Quadrotor modelling and control".

[2] R. C. Avram, X. Zhang and J. Muse 2018 "Nonlinear Adaptive Fault-Tolerant Quadrotor Altitude and Attitude Tracking With Multiple Actuator Faults," in IEEE Transactions on Control Systems Technology.

[3] Z. He and L. Zhao 2017 "Internal model control /backstepping sliding model control for quadrotor trajectory tracking," in 2017 IEEE 2nd Information Technology, Networking, Electronic and Automation Control Conference (ITNEC). 
[4] M. Farhan, A. Bhatti, W. Kamal and I. Yousafzai 2017 "Sliding mode based MIMO control of autonomous underwater vehicle," in Control Conference (ASCC), 2017 11th Asian, Gold Coast, Australia, Australia.

[5] M. Tran, J. Binns, S. Chai, A. Forrest and H. Nguyen 2017 "Optimal control of an autonomous underwater vehicle equipped with the collective and cyclic pitch propeller," in 2017 11th Asian Control Conference (ASCC).

[6] S. C. Martin and L. L. Whitcomb 2018 "Nonlinear Model-Based Tracking Control of Underwater Vehicles With Three Degree-of-Freedom Fully Coupled Dynamical Plant Models: Theory and Experimental Evaluation," in IEEE Transactions on Control Systems Technology.

[7] G. Ferri, A. Munafò and K. D. LePage 2018 "An Autonomous Underwater Vehicle Data-Driven Control Strategy for Target Tracking," in IEEE Journal of Oceanic Engineering.

[8] Z. Sun, Q. Chen, Y. Nie, D. Liu and H. He, "Ribbon Model based path tracking method for autonomous land vehicle," in Intelligent Robots and Systems (IROS), 2012 IEEE/RSJ International Conference on, Vilamoura, Portugal , 2012.

[9] Q. Xu, X. Li, B. Li, X. Song and Z. Cai, "A Reliable Hybrid Positioning Methodology for Land Vehicles Using Low-Cost Sensors," in IEEE Transactions on Intelligent Transportation Systems, 2015.

[10] Wahyudi and Ngatelan, "Design of multi-sensor IMU for land vehicle," in Information Technology, Computer, and Electrical Engineering (ICITACEE), 2015 2nd International Conference on, Semarang, Indonesia, 2015.

[11] G. D. S. Lee, K. S. Lee, H. G. Park and M. H. Lee, "Optimal path planning with holonomic mobile robot using localization vision sensors," in ICCAS 2010, Gyeonggi-do, South Korea, 2010.

[12] S. K. P and B. S, "Position control of a flexible manipulator using a new nonlinear self tuning PID controller," in IEEE/CAA Journal of Automatica Sinica , 2018.

[13] M. Zogg, "Global Positioning System, Standard Positioning System Service, Signal Specification, 2nd Edition," 1995. 